\title{
Incorporating a Vegetation Index into a Soil Moisture Retrieval Model - Results from Convair-580 SAR Data
}

\author{
Millie Sikdar $^{1}$, Scott MacIntosh ${ }^{1}$, Ian Cumming ${ }^{1}$ and Brian Brisco ${ }^{2}$ \\ ${ }^{1}$ Department of Electrical and Computer Engineering \\ The University of British Columbia \\ Vancouver, BC, Canada \\ ${ }^{2}$ Noetix Research \\ 403-265 Carling Avenue \\ Ottawa, ON, Canada
}

\begin{abstract}
A flexible method of introducing a SAR vegetation index into the Dubois model for soil moisture retrieval using polarimetric SAR data is introduced. Based on the vegetation sensitivity at each pixel, the vegetation index is incorporated on a pixel by pixel basis using the water cloud model. An approach for minimizing the need for ground measurements, by remotely estimating the parameters required for the vegetation index, is suggested. The proposed model is applied to CV-580 airborne SAR data and the vegetation correction was found to reduce the rms error in the model.
\end{abstract}

\section{INTRODUCTION}

Future spaceborne SAR systems such as the RADARSAT-2, ALOS PALSAR, EMISAR and HYDROS promise to provide large quantities of polarimetric SAR data, thus leading to the feasibility of global soil moisture estimation on a global scale. There is significant interest in the ability to remotely estimate soil moisture content due to the pivotal role it plays in understanding agricultural and ecological processes and the nature of global change. Consequently, it is important to develop models for accurate soil moisture retrieval from polarimetric SAR data.

Models for soil moisture retrieval were originally developed for bare soil areas. However, it is important to be able to estimate soil moisture in vegetated regions as well. While the vegetation effects have been successfully introduced into a few of the existing soil moisture retrieval algorithms, more work has to be done to improve the effectiveness of using a vegetation index in Dubois' empirical model [1].

This paper primarily focuses on improving the method of introducing vegetation correction into the Dubois model. In addition, the concern of preserving the essence of "remote sensing" by estimating the vegetation parameters remotely rather than using ground based measurements is addressed here and a possible solution is discussed.

\section{BACKGROUND}

Most of the models developed for soil moisture estimation in both bare and vegetated regions were based on of inverting direct models (backscatter as a function of soil moisture) in order to estimate the surface parameters from the given radar measurements. These empirical models were generally formulated using radiometer or SAR measurements. It is important to note that under vegetated conditions, the radiometric retrieval algorithms were found to provide more accurate soil moisture estimates as compared to the algorithms based on SAR data. However, one of the major drawbacks associated with radiometers is their low spatial resolution. Even a highresolution radiometer such as the AVHRR (Advanced Very High Resolution Radiometer) provides a resolution of only $1.1 \mathrm{~km}$. In comparison, SAR data has high spatial resolution and provides fine-scale vegetation information within the lower resolution radiometer footprint [3]. As a result, significant interest lies in developing models capable of using SAR data for accurate estimation in regions with considerable amounts of vegetation, especially when a radiometer is an adjunct instrument.

A discussion of the different types of algorithms for soil moisture retrieval and their drawbacks can be found in [2]. A common approach used for developing models for vegetated conditions is to introduce vegetation effects into an existing bare-soil model. The present work is based on a similar concept and the empirical model of Dubois et al. [1] is selected as the most suitable choice for our application [2]. The Dubois model was developed using radiometer-scatterometer data, making it more suitable for vegetated regions as compared to other models such as the Oh [4] and the Shi models [5].

\section{Modeling SoIL Moisture}

The modified empirical model developed by incorporating vegetation effects into the existing Dubois model was found to yield a significant increase in the estimation accuracy in vegetated regions [2]. However, the model is unable to achieve the degree of accuracy obtained by the vegetation model proposed by Bindlish et al. [6].

The primary goal of the present work is to improve upon the methodology used to include the contribution of vegetation into the Dubois model. An attempt is also made to propose a systematic approach for remotely calculating vegetation parameters required in the vegetation index, thus avoiding tedious ground measurements. 


\section{A. Incorporating a vegetation index into the existing model}

The Water-Cloud model proposed by Attema \& Ulaby in 1978 [7] provides a simple approach to include the contribution of the vegetation as well as the soil in the backscattering coefficient. According to the model, the total power scattered at a co-polarized channel $\mathrm{pp}, \sigma_{\mathrm{pp}}^{\mathrm{o}}$, is the incoherent sum of contribution of the vegetation, $\sigma_{\mathrm{veg}}^{\mathrm{O}}$, and that of the underlying soil, $\sigma_{\text {soil }}^{\mathrm{O}}$, which is attenuated by the vegetation layer. In order to include the effects of orientation and geometry of the vegetation, Bindlish $\&$ Barros subsequently modified this model by introducing the vegetation correlation length, $\alpha$ [6]. The modified water-cloud model thus expresses $\sigma_{\mathrm{pp}}^{\mathrm{o}}$ as:

$$
\begin{aligned}
& \sigma_{\mathrm{pp}}^{\mathrm{o}}=\sigma_{\mathrm{veg}}^{\mathrm{o} *}+\tau^{2} \sigma_{\mathrm{soil}}^{\mathrm{o}} \\
& \text { with } \quad \tau^{2}=e^{\left\{-2 b \mathrm{~W}_{\mathrm{c}} \sec \theta\right\}} \\
& \sigma_{\mathrm{veg}}^{\mathrm{o} *}=\sigma_{\mathrm{veg}}^{\mathrm{o}}\left(1-e^{-\alpha}\right) \\
& \text { and } \quad \sigma_{\mathrm{veg}}^{\mathrm{o}}=A m_{v} \cos \theta\left(1-\tau^{2}\right)
\end{aligned}
$$

where $\sigma_{\text {veg }}^{\text {o* }}$ is the backscatter contribution of the vegetation corrected for the effects of orientation and geometry of the canopy. The parameter, $\alpha$, is a function of the average distance between vegetation canopies within a pixel, $m_{v}$ is the volumetric soil moisture content, $\tau^{2}$ is the two-way vegetation transmissivity, $W_{c}$ is the vegetation water content in $\mathrm{kg} / \mathrm{m}^{2}$ and $\theta$ is the radar incidence angle.

The vegetation-dependent parameters, $A$ and $b$, determined from experimental observations [8], represent the vegetation scattering and the vegetation attenuation, respectively. Also, other vegetation parameters such as $W_{c}$ and $\alpha$ are measured directly at the ground. Consequently, this indicates that the transmissivity, $\tau^{2}$, and all the parameters derived from the parameters, as given in (1) to (4), rely on ground based measurements. This concern is addressed in the next section.

The above modified water cloud model shows that a considerable number of factors are taken into account in order to determine a sufficiently correct estimate of the vegetation backscatter, $\sigma_{\mathrm{veg}}^{\mathrm{o}}$.

In the modified empirical model of [2], the soil backscatter, $\sigma_{\text {soil }}^{\mathrm{o}}$, is computed by a Least Mean Squares (LMS) regression between the measured backscatter and the volumetric soil moisture [9]. The regression analysis expresses the soil backscatter as a linear function of the soil moisture content:

$$
\sigma_{\text {soil }}^{\mathrm{o}}=C+D m_{v} \quad \mathrm{~dB}
$$

Here, the Dubois model is used to obtain an initial estimate of the soil moisture content, $m_{v}$. The use of the Dubois model itself at an initial stage to compute the soil backscatter, which is subsequently used in the water-cloud model, leads to a significant probability that errors are introduced into the modified model given in [2].

In the present work, Dobson's model for the soil backscatter [10] is employed to compute $\sigma_{\text {soil }}^{\mathrm{o}}$ in conjunction with the linear regression analysis. According to Dobson and Ulaby, the soil backscatter can be given by the general form:

$$
\sigma_{\text {soil }}^{\mathrm{o}}=4(k s)^{2}(k L)^{2} \cos ^{4} \theta e^{\left\{-(k L \sin \theta)^{2}\right\}}\left|\Gamma_{\mathrm{o}}\right|^{2}
$$

where $k=2 \pi / \lambda$ is the radar wavenumber, $s$ is the surface roughness, $L$ is the surface correlation length and $\theta$ is the incidence angle. The Fresnel reflectivity, $\Gamma_{o}$, can be obtained from the dielectric constant, $\epsilon^{\prime}$, using [4]:

$$
\Gamma_{0}=\left|\frac{1-\sqrt{\epsilon^{\prime}}}{1+\sqrt{\epsilon^{\prime}}}\right|
$$

The original Dubois model is initially implemented to obtain an estimate of the surface roughness and the dielectric constant required in (6) and (7). The surface correlation length is obtained from ground measurements.

In the modified empirical model [2], the vegetation effects were introduced into the existing model only if the average cross-polarization ratio for a particular site was greater than $-11 \mathrm{~dB}$. Based on this train of thought, the adaptive nature of the model can be improved by evaluating the vegetation sensitivity on a pixel by pixel basis.

The vegetation-covered soil moisture sensitivity, $S_{v}$, was defined by Ulaby et al. [11] as:

$$
S_{v}=\frac{S_{B}}{\left(1+\frac{\sigma_{\mathrm{veg}}^{\text {o* }}}{\tau^{2} \sigma_{\mathrm{soil}}^{\mathrm{o}}}\right)}
$$

where the sensitivity of bare soil, $S_{B}$, is given as:

$$
S_{B}=4.34 D
$$

The values of $\sigma_{\text {veg }}^{\mathrm{o}}, \tau^{2}$ and $\sigma_{\text {soil }}^{\mathrm{o}}$ are obtained using (3), (2) and (6), respectively. The coefficient, $D$, is determined from the LMS regression analysis of (5).

By examining the limits of $S_{v}$ as function of the ratio inside the brackets in (8), it is evident that:

$$
\begin{aligned}
S_{v} & \simeq 0, \quad \text { for } \tau^{2} \sigma_{\text {soil }}^{\mathrm{o}} \ll \sigma_{\mathrm{veg}}^{\mathrm{o}} \\
\text { and } \quad S_{v} & \simeq S_{B}, \quad \text { for } \tau^{2} \sigma_{\text {soil }}^{\mathrm{o}} \gg \sigma_{\mathrm{veg}}^{\mathrm{o}}
\end{aligned}
$$

The first limit, $S_{v} \simeq 0$, represents the case where the vegetation completely masks the soil, while the second limit, $S_{v} \simeq$ $S_{B}$, represents the situation where backscatter is dominated by the soil contribution. Consequently, pixels for which $S_{v}<S_{B}$ may be considered as vegetated and vegetation correction is applied only to these pixels.

\section{B. Remote estimation of vegetation parameters}

In addition to the concern for higher estimation accuracy in regions with larger amounts of vegetation, it is also desired that a retrieval algorithm should require minimum groundmeasurable parameters, instead it should rely on determining these parameters remotely.

Ulaby \& El-Rayes suggested a dielectric model for the vegetation, where the vegetation transmissivity, $\tau$, could be calculated as [12]: $\quad \tau=4 \pi(d / \lambda) \operatorname{Im}\left[\sqrt{\epsilon_{v}}\right]$

where $d$ is the thickness of the layer, $\lambda$ is the radar wavelength and $\epsilon_{v}$ is the vegetation dielectric constant determined by:

$$
\begin{aligned}
\epsilon_{v}=\epsilon_{r} & +v_{\mathrm{fw}} \\
+ & v_{b}\left[2.9+\frac{75}{1+\mathrm{j} f / 18}-\mathrm{j} \frac{18 \sigma}{f}\right] \\
& {\left[\frac{55}{1+(\mathrm{j} f / 18)^{0.5}}\right] }
\end{aligned}
$$


In the above relation, $\epsilon_{r}$ is a non-dispersive residual component, $f$ is the frequency in $\mathrm{GHz}, \sigma$ is the ionic conductivity in siemens per meter, and $v_{\mathrm{fw}}$ and $v_{b}$ are the volume fractions of free water and bulk vegetation-bound water, respectively.

According to regression curves obtained from a number of experimental data sets, it was concluded that the parameters in (13) can be computed by the following equations:

$$
\begin{aligned}
m_{v} & =m_{g} \rho /\left[1-m_{g}(1-\rho)\right] \\
\epsilon_{r} & =1.7-0.74 m_{g}+6.16 m_{g}^{2} \\
v_{\mathrm{fw}} & =m_{g}\left(0.55 m_{g}-0.076\right) \\
v_{b} & =4.64 m_{g}^{2} /\left(1+7.36 m_{g}^{2}\right) \\
\sigma & =1.27
\end{aligned}
$$

where $m_{v}$ and $m_{g}$ are the volumetric and gravimetric soil moisture contents, respectively. Thus, by going backwards from (18) to (12), the transmissivity, $\tau^{2}$ can be determined without the need of the vegetation parameters, $A$ and $b$.

As compared to (1) to (4), the only ground measurements required in the above dielectric model are the vegetation thickness, $d$, and the soil bulk density, $\rho$. This is a better choice of model, since the parameters, $A$ and $b$, are found from regression analysis of suitable, available ground measurements.

The vegetation water content can be determined remotely by using the normalized vegetation difference index (NDVI). NDVI is a measure of the vegetative cover and is generally calculated from reflectances measured in the visible and near infrared channels of hyperspectral imagery. A non-linear relation between the NDVI and $W_{c}$ is derived in [13] by optimizing a polynomial function:

$$
\begin{aligned}
& \text { If } \mathrm{NDVI} \leq 0.5 \\
& \quad W_{c}\left(\mathrm{~kg} / \mathrm{m}^{3}\right)=1.9134(\mathrm{NDVI})^{2}-0.3125(\mathrm{NDVI}) \\
& \text { If } \mathrm{NDVI}>0.5 \\
& \quad W_{c}\left(\mathrm{~kg} / \mathrm{m}^{3}\right)=4.2857(\mathrm{NDVI})-1.5429
\end{aligned}
$$

Dubois et al. provide a regression curve between the crosspolarization ratio for L-band data and the NDVI [1]. As a result, for cases where L-band data is available, the NDVI can be computed using this alternate method; else hyperspectral imagery can be used.

\section{IMPLEMENTATION}

The augmented algorithm proposed in Section III-A is applied to C-band, Convair-580 airborne SAR data acquired during a polarimetric data collection campaign in Ottawa. Two different passes (L2P3 and L3P2) of data were collected over the same agricultural area on June 26 and July 19, 2001. The test sites in the image, shown in Fig. 1, include four different fields comprising of corn, wheat and soybean.

Both data sets include ground measurements of the soil moisture content, surface roughness, plant height and the vegetation water content. However, the vegetation parameters, $A, b$ and $\alpha$, for each vegetation type are not specified in the ground truth. Furthermore, the vegetation thickness, $d$, and the soil bulk density, $\rho$, for each field have not been specified, thus limiting the scope for the computation of the vegetation transmissivity, $\tau^{2}$. Instead, the vegetation parameters derived previously in [6] are used. This assumption is based on the overall similarity of the vegetation types used in [6] to those in our current data set. Table I summarizes the vegetation parameters that are assumed for each field.

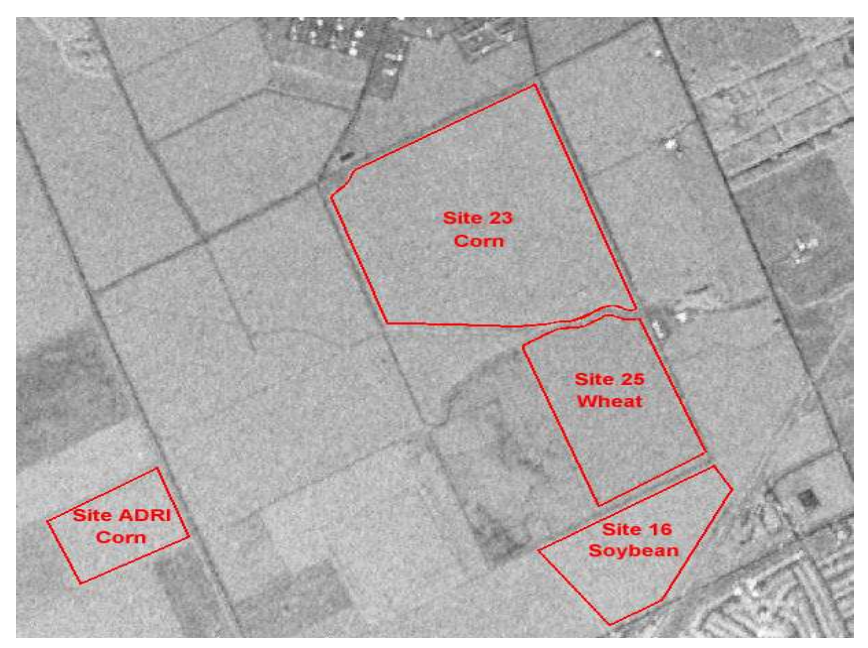

Fig. 1. Location of study sites used for the $2001 \mathrm{CV}-580$ field campaign

Since the CV-580 data is C-band and hyperspectral data is not available, the NDVI for a certain field or pixel cannot be computed. As a result, it is not possible to apply the method suggested in Section III-B to remotely determine $W_{c}$ for the present data set. Further, it is important to note that the ground report does not specify the correlation length, $L$ required in (6) and a value is estimated for the each crop type that gives the best vegetation correction to the data.

TABLE I

Site Characterization For Polarimetric Data Collection CAmpaign in OtTaWa, 2001. Source: [14], [8], [6]

\begin{tabular}{|c|c|c|c|c|c|}
\hline Site & Land Cover & $\mathrm{W}_{\mathrm{c}}\left(\mathrm{kg} / \mathrm{m}^{2}\right)$ & $A$ & $b$ & $\alpha$ \\
\hline 25 & Wheat & 2.31 & 0.0018 & 0.138 & 0.96 \\
23 & Corn & 1.25 & 0.0012 & 0.091 & 2.12 \\
16 & Soyabean & 2.31 & 0.0014 & 0.084 & 0.979 \\
ADRI & Corn & 0.36 & 0.0012 & 0.091 & 2.12 \\
\hline
\end{tabular}

As in [2], a regression analysis is initially performed on the measured VV backscatter, $\sigma_{\mathrm{vv}}^{\mathrm{O}}$, and the soil moisture content, $m_{v}$, estimated by the original Dubois model. The derived linear relationship is further used to determine the sensitivity of bare soil, $S_{B}$, and subsequently the vegetation sensitivity, $S_{v}$, as given in (8) and (9). Also, the application of the Dubois model provides the surface roughness, $s$, and Fresnel reflectivity, $\Gamma_{0}$, required to compute $\sigma_{\text {soil }}^{\mathrm{o}}$ at a later stage.

Based on the experimental observations in [2], the vegetation effects are introduced only into the $\mathrm{VV}$ backscatter $(\mathrm{HH}$ is used in the Dubois model without a vegetation correction). The vegetation backscatter, $\sigma_{\text {veg }}^{\text {o* }}$, and the soil backscatter, $\sigma_{\text {soil }}^{\mathrm{o}}$, for each pixel are obtained from (3) and (6). Further, 
the modified empirical model is now applied on a pixel basis only for the condition when the vegetation sensitivity, $S_{v}$, at a certain pixel is less than $S_{B}$. The vegetation corrected VV backscatter, $\sigma_{\mathrm{vv}}^{\circ}$, determined using the water-cloud model is finally used in Dubois' inversion model to obtain the corrected estimates of the volumetric soil moisture, $m_{v}$.

\section{RESUlTS}

The plots of the estimated values of volumetric soil moisture for the original empirical model and for the proposed algorithm with vegetation correction are shown in Figs. 2 and 3 , respectively. The RMS deviations from the measured values have been computed to estimate the accuracy of each model. A $45^{\circ}$ line is shown to indicate perfect model correspondence.

A visual comparison of the plots indicates that the introduction of the vegetation index improves the accuracy of the estimation, as compared with the original bare soil model.

At this point in time, two of the fields in the July 19 data are giving anomalous results from the vegetation model, and have been removed from the graph. They are affected by the method of computing dielectric constant. We are presently trying to resolve this. Additional results will be given at the conference and posted on: http://sar.ece.ubc.ca/papers/

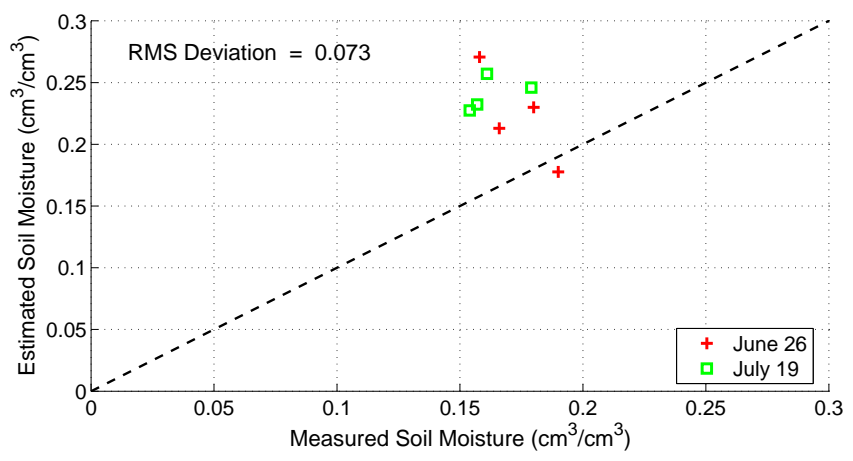

Fig. 2. Soil moisture results for the Dubois (bare soil) model

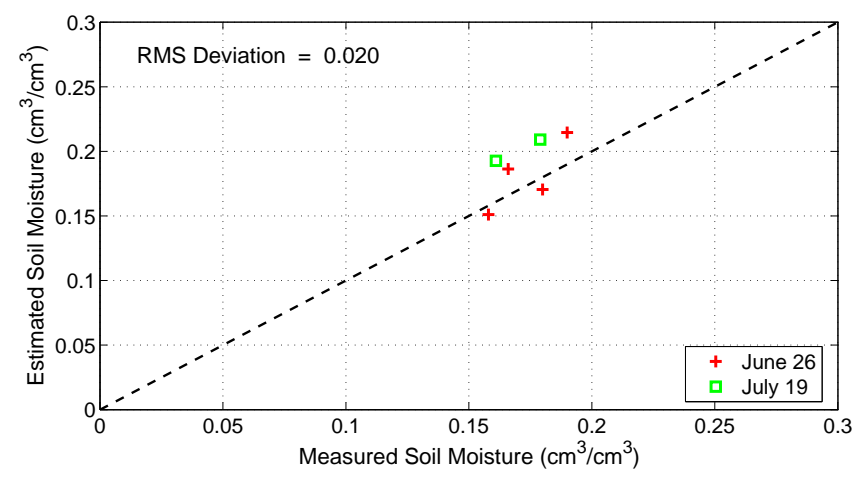

Fig. 3. Soil moisture results with vegetation correction

\section{SUMMARY}

An improved methodology for incorporating a vegetation index into the Dubois model is presented. The modified model allows the vegetation correction to be applied on a per-pixel basis by evaluating the vegetation sensitivity at each pixel and subsequently applying the vegetation correction only at pixels with a considerable amount of vegetation. Further, an approach for the remote estimation of vegetation parameters is discussed.

As seen in the results, the proposed model improves the soil moisture estimation compared to the original Dubois model. Note that the vegetation parameters were not all available in the ground truth, hence, they were selected from prior experiments. Also, the correlation length had to be determined by an arbitrary fitting. As a result, it is difficult to verify the accuracy of the model in absolute terms. However, the model does provide the structure and degrees of freedom to give suitable vegetation-corrected results when a plausible set of ground measurements are available.

\section{ACKNOWLEDGEMENTS}

The authors would like to thank Dr. E. Pattey of Agriculture and Agri-Food Canada for the Convair-580 data and NSERC and MacDonald Dettwiler for research funds.

\section{REFERENCES}

[1] P. C. Dubois, J. van Zyl, and E. T. Engman, Measuring Soil Moisture with Imaging Radars, IEEE Trans. Geosci. Rem. Sensing, 1995, Vol. 33, No. 4, pp. 915-927

[2] M. Sikdar and, I. G. Cumming, A Modified Empirical Model for Soil Moisture Estimation in Vegetated Areas Using SAR Data, Proc. IGARSS '04, 2004, Anchorage, Vol. 2, pp. 803-806

[3] D. Entekhabi, et al., The Hydrosphere State (HYDROS) Mission Concept: An Earth System Pathfinder for Global Mapping of Soil Moisture and Land Freeze/Thaw, IEEE Trans. Geosci. Rem. Sensing, 2004, Vol. 42, No. 10, pp. 2184-2195

[4] Y. Oh, K. Sarabandi, and F. T. Ulaby, An Empirical Model and an Inversion Technique for Radar Scattering from Bare Soil Surfaces, IEEE Trans. Geosci. Rem. Sensing, 1992, Vol. 30, No. 2, pp. 370-381

[5] J. C. Shi, J. Wang, A. Hsu, P. E. O’Neill, and E. T. Engman, Estimation of Bare Surface Soil Moisture and Surface Roughness Parameter Using L-Band SAR Image Data, IEEE Trans. in Geosci. Rem. Sensing, 1997, Vol. 35, No. 5, pp. 1254-1266

[6] R. Bindlish, and A. P. Barros, Parameterization of vegetation backscatter in radar-based soil moisture estimation, Rem. Sensing of Env, 2001, Vol. 76, pp. 130-137

[7] E. P. W. Attema, and F. T. Ulaby, Vegetation Modeled as a Water Cloud, Radio Science, 1978, Vol. 13, No. 2, pp. 357-364

[8] T. J. Jackson, and T. J. Schmugge, Vegetation Effects on the Microwave Emission of Soils, Rem. Sensing of Env, 1991, Vol. 36, pp. 203-212

[9] F. T. Ulaby, R. K. Moore, and A. K. Fung, Microwave Remote Sensing: Active and Passive, 1986, Vol III, Artech House, pp. 915-927

[10] M. C. Dobson, and F. T. Ulaby, Preliminary Evaluation of the SIR-B response to Soil Moisture, Surface Roughness, and Crop Canopy Cover, IEEE Trans. Geosci. Rem. Sensing, 1986, Vol. GE-24, No.4, pp. 517-526

[11] F. T. Ulaby, G. D. Bradley, and M. C. Dobson, Microwave Backscatter Dependence on Surface Roughness, Soil Moisture and Soil Texture, PartII:Vegetation-Covered Soil, IEEE Trans. Geosci. Elect., 1979, Vol. GE17 , pp. $33-40$

[12] F. T. Ulaby, and M. A. El-Rayes, Microwave Dielectric Spectrum of Vegetation - Part II: Dual Dispersion Model, IEEE Trans. Geosci. Rem. Sensing, 1987, Vol. GE-25, No. 5, pp. 550-557

[13] T. J. Jackson, et al., Soil Moisture Mapping at Regional Scales Using Microwave Radiometry: The Southern Great Plains Hydrology Experiment, IEEE Trans. Geosci. Rem. Sens., 1999, Vol. 37, No. 5, pp. 2136-2151

[14] M. Hinther, et al., Report on Ground Truthing Data Documentation for the CASI and CV-580 Polarmetric Data Collection Campaign in Ottawa for the 2001 Growing Season, Contributors: ECORC, CCRS, Noetix Research, McGill University 